\title{
Asociación entre morbilidad neonatal y desarrollo en pretérminos a la edad escolar
}

\author{
1. Profesor de Neonatología. \\ 2. Licenciado en Psicología. \\ 3. Auxiliar de enfermería CUP. \\ 4. Pediatra. \\ 5. Fonoaudióloga. \\ 6. Oftalmólogo \\ 7. Departamento de Biofísica.
}

MIGUEL MARTELL' ${ }^{1}$, MARISA BURGUEÑO², GRACIELA ARBÓN ${ }^{3}$, MARINA WEINBERGER ${ }^{4}$, CECILIA BALBI $^{4}$, ALICIA MUNYO 5 , LEONORA MARTINOTTI ${ }^{6}$, SILVIO MURILLO ${ }^{6}$, RUTH KESHISHIAN ${ }^{4}$, ANDRÉS POMI $^{4}$, RAFAEL ALONSO$^{7}$

\begin{abstract}
Association between morbidity and development in preterm newborn to school age

The aim of the study is the relationship between the morbility of very low birthweight newborns which required neonatal intensive care and their cognitive abilities during school. Material and Method: This is a prospective cohort reconstruction study with a sample of 18 newborns whose birthweight was lower than $1250 \mathrm{~g}$. It was compared with a control group (term newborns with adequate birthweight), paired by their socioeconomic status. During the neonatal period in the intensive care unit morbility and neonatal severity were assessed using SNAP and NTISS from the first day with weekly controls, until delivery day. Two postnatal evaluations were done: pre-school assessment (between 3 and 4 years old) and at school age (between 8 and 9 years old). In the first evaluation the Denver scale was used to evaluate their neurodevelopment. A health classification system which can detect the health state of a child (MASH) was also used. During school-age the WISC-III scale which evaluates cognitive habilitéis was applied. In both periods weigth, height and craneal perimeter were measured. The relationship between the number of pathologies and cognitive scale was studied with a linear regression. Results: The average cognitive capacity (CIG) was $87,5 \pm 14,3$ in the preterm group and $104,3 \pm 12,18$ in the control group, showing a significant difference $(\mathrm{p}<0,003)$. The most frequent pathology was sepsis $(47,8 \%)$. Pathology decreases cognitive score in 10 points (CI $95 \%$ = between 5 and 14), with a determinant coeficient: $r 2=0,60(p<0,05)$. Children who had three or more diseases developed severe neurodevelopment retardation. The severity of illness was significantly higher between 3 and 4 weeks of life. Conclusions: We found a significant relationship between neonatal complications and school outcomes in very low birth weight $(<1.250 \mathrm{~g})$.The newborn care in the intensive care units must aim at avoiding these complications, especially infectious diseases. Neonatal care implies a big responsibility for tose who are in charge of the infrastructure and
\end{abstract}


supervisision of the well-fucntioning of the units. Health carers should bare in mind that every complication Hill affect between 5 and 14 points the cognitive capacity at school.

(Key words: Infant, very low birth weight infant, premature, morbidity, child development, cognition).

Arch Pediatr Urug 2007; 78(2): 99-109

\section{RESUMEN}

El objetivo del presente trabajo es estudiar la asociación entre la morbilidad en neonatos nacidos con un peso menor de 1,250 gramos que requirieron cuidado intensivo neonatal y la capacidad cognitiva a la edad escolar. Material y Método: Es un estudio prospectivo de reconstrucción de cohortes en una muestra de 18 neonatos cuyo peso al nacimiento fue menor de 1250 gramos. Se comparan con un grupo control de niños nacidos de término y peso adecuado, pareados por medio socioeconómico. Durante la internación neonatal se registró la morbilidad y la gravedad neonatal evaluada por el SNAP y NTISS que se realizó al ingreso y semanalmente hasta el alta. Se realizaron dos evaluaciones posnatales: una en el período preescolar, entre los tres y cuatro años, y la otra en edad escolar, entre ocho y nueve años. En la primera evaluación se valoró el desarrollo con la escala de Denver y un sistema de atributos que mide el estado de salud del niño (MASH). A la edad escolar se usó la escala de WISC-III para medir la capacidad cognitiva. En ambos controles se evaluó el crecimiento a través del peso, talla y perímetro craneano. La asociación entre el número de morbilidades y la escala cognitiva se estudió usando una regresión lineal simple. Resultados: El promedio de la capacidad cognitiva (CIG) fue de 87,5 $\pm 14,3$ en el grupo de pretérmino y de $104,3 \pm 12,18$ para el grupo control. Esta diferencia fue significativa $(\mathrm{p}<0,003)$. La morbilidad más frecuente fue la sepsis $(47,8 \%)$. Se encontró que cada morbilidad disminuye el puntaje cognitivo en 10 puntos (IC entre 5 y 14), con un coeficiente de determinación $\mathrm{r} 2=0,60(\mathrm{p}<0,05)$. Los niños que tuvieron tres o más morbilidades presentaron retardo severo. La gravedad de éstos fue significativamente mayor entre las tres y cuatro semanas y el crecimiento menor que los que tuvieron evolución normal. Conclusiones: Estos resultados muestran la contribución de las complicaciones neonatales en la unidad de terapia intensiva con las alteraciones del desarrollo en la edad escolar. Se deben extremar los cuidados de estos niños para evitar las complicaciones, especialmente la infección. El cuidado neonatal implica una gran responsabilidad de las personas que se encargan del cuidado directo y de los que mantienen la infraestructura. Cada complicación en la unidad de cuidados intensivos disminuirá entre 5 y 10 puntos la capacidad cognitiva.

(Palabras clave: Recién nacido de muy bajo peso, prematuro, morbilidad, desarrollo infantil, cognición). Arch Pediatr Urug 2007; 78(2): 99-109

ESTE TRABAJO LO PUEDE ENCONTRAR EN EXTENSO EN WWW.SCIELO.CL 